\title{
Information use shapes the dynamics of range expansions into environmental gradients
}

\author{
Emanuel A. Fronhofer ${ }^{1,2, *}$, Nicolai Nitsche ${ }^{3}$ and Florian Altermatt ${ }^{1,2}$
}

1. Eawag: Swiss Federal Institute of Aquatic Science and Technology, Department of Aquatic Ecology, Überlandstrasse 133, CH-8600 Dübendorf, Switzerland

2. Department of Evolutionary Biology and Environmental Studies, University of Zurich, Winterthurerstrasse 190, CH-8057 Zürich, Switzerland

3. Ecology, Department of Biology, University of Konstanz, Universitätsstrasse 10, D-78457 Konstanz, Germany

Keywords: range expansion, biological invasion, environmental gradient, information use, dispersal

Running title: Informed dispersal and range dynamics

Counts: Abstract: 260 words; main text: ca. 5050 words; no. references: 38.

Correspondence Details

Emanuel A. Fronhofer

Eawag: Swiss Federal Institute of Aquatic Science and Technology

Department of Aquatic Ecology

Überlandstrasse 133

CH-8600 Dübendorf

phone: +41587655143

email: emanuel.fronhofer@eawag.ch

This document is the accepted manuscript version of the following article:

Fronhofer, E. A., Nitsche, N., \& Altermatt, F. (2017). Information use shapes the dynamics of range expansions into environmental gradients. Global Ecology and Biogeography, 26(4), 400-411. https://doi.org/10.1111/geb. 12547 
2 Aim Globally, geographic distributions of species are dynamic and strongly influenced by dispersal. At 3 the same time, range dynamics feed back and may select for increased dispersal at expanding range fronts.

4 This interplay between macroecological and evolutionary dynamics almost universally happens across en5 vironmental gradients and such gradients can directly impact the fitness of organisms due to the match or 6 mismatch between an individual's environmental optimum and the current conditions along the gradient.

7 Importantly, gradients also provide individuals with information on the environmental changes because

8 dispersing individuals may sense whether environmental conditions improve or deteriorate. However, the 9 organisms' ability to use this information on the environment and to subsequently adjust dispersal decisions plastically, that is, deciding to further disperse into the gradient or not, has been largely ignored and 1 the macroecological consequences remain unclear. We here aim at demonstrating the impact of informed 2 dispersal on the eco-evolutionary dynamics of ranges.

13 Methods We used individual-based simulations and controlled experiments in replicated microcosm 14 landscapes. Range expansions of the protist model organism Tetrahymena were tracked using video 15 recording and analysis.

16 Results We show that information on environmental gradients severely impacted range dynamics and 7 inverted the spatial distribution of population densities in comparison to controls where this information was not provided. Additionally, using information on gradients prevented evolutionary changes in dispersal rates and an acceleration of range expansions.

Main conclusions We demonstrate the strong impact of informed dispersal and subsequent behavioral changes on range dynamics in environmental gradients. More generally, our findings highlight the importance of informed dispersal for spatial ecological and evolutionary dynamics. 


\section{Introduction}

The capacity of organisms to spread in space and to expand their range into new habitat is crucial for their long-term fitness, especially in the context of current global environmental and climatic changes (Hill et al., 1999; Parmesan et al., 1999; Parmesan \& Yohe, 2003; Kelly \& Goulden, 2008). The fundamental and applied relevance of range expansions and biological invasions resulted in extensive theoretical work predicting range dynamics (Hastings et al., 2005; Holt et al., 2005; Burton et al., 2010; Dytham, 2009; Holt \& Barfield, 2011; Perkins et al., 2013; Williams et al., 2016). To date, however, our empirical understanding of range dynamics is mostly based on case studies of range shifts and invasions with little experimental validation or manipulation (e.g., Thomas et al., 2001; Phillips et al., 2006; Lombaert et al., 2014). The few studies that experimentally track replicated range expansions are either limited by the short time frames considered (Melbourne \& Hastings, 2009; Giometto et al., 2014), preventing potentially important evolutionary changes to occur, or by the unrealistic assumption that range expansions occur into uniform habitat (Fronhofer \& Altermatt, 2015).

Realistically, all range expansions are limited by the heterogeneity of landscapes and the universally present gradients in environmental conditions, such as temperature or humidity. The importance of gradients as such for species ranges has been explored previously: gradients may, for instance, impact dispersal evolution (Kubisch et al., 2014), lead to stable range borders and even range contractions after the expansion phase (Kubisch et al., 2010, 2016), gradients are often associated with the distribution of population densities throughout the range (Sagarin et al., 2006; Sexton et al., 2009) and the relevance of species interactions for range dynamics is hypothesized to be tightly linked to the harshness of environmental gradients (Louthan et al., 2015). However, these works usually ignore that environmental gradients have a two-fold effect on organisms: 1) Gradients have a direct, fitness-relevant effect due to the mismatch between local conditions and the individuals' environmental optimum. 2) Furthermore, gradients have indirect effects mediated by the information the gradient conveys to dispersing organisms. Dispersers may be able to sample information on the environmental gradient, relate it to their level of (mal)adaptation to local conditions and finally make informed dispersal decisions on whether to disperse further into the gradient or not. If we take the example of a temperature gradient, dispersing individuals may be able to sense differences in temperature between patches as they move through the landscape and, if the local temperature gets too high or too low relative to their temperature optimum, they can decide not to disperse further into the gradient, or even to disperse backwards. While the relevance of information use for making dispersal decisions and subsequent consequences for spatial dynamics has been recognized in general (Clobert et al., 2009), the consequences of informed dispersal for macroecological dynamics, such as species range shifts, remain under-appreciated. 
Here, we theoretically and experimentally test the role of environmental gradients for the dynamics of range expansions taking into account the two-fold effect of environmental gradients discussed above. We use an individual-based model to predict ecological and evolutionary dynamics in three range expansion scenarios: Firstly ("control"), we model the range expansion of individuals into a previously empty linear landscape of interconnected patches. Secondly, we include a scenario analogous to the first, but where the landscape is characterized by a linear gradient of increasing local mortality that affects the spreading organisms' fitness without providing information on the spatial change in mortality ("gradient"). Finally, we contrast these two scenarios with a range expansion into a mortality gradient that provides information on the changes in mortality and individuals use this information to make optimal dispersal decision plastically ("gradient \& information").

We tested our theoretical predictions using experimental evolution and replicated linear microcosm landscapes, which were invaded by the ciliate model organism Tetrahymena pyriformis (Altermatt et al., 2015). The landscapes allowed for active dispersal and included the three scenarios detailed above: control, gradient as well as gradient \& information.

We predict that range expansions in the control scenario lead to the evolution of increased dispersal at the range front (Phillips et al., 2006; Fronhofer \& Altermatt, 2015). The mortality gradient in the second scenario should lead to a reduction in range expansion speed and, ultimately, to the establishment of a stable range border due to the ecological effect of the mortality gradient on local population dynamics and the evolutionary effect selecting against dispersal (Kubisch et al., 2014). Finally, the availability of information should provide organisms with the opportunity to make informed and plastic dispersal decisions and thereby not to disperse into areas characterized by high local mortalities.

\section{Materials and Methods}

\section{${ }_{78}$ Numerical analyses}

\section{General overview}

80 We developed a stochastic, individual-based simulation model (Burton et al., 2010; Kubisch et al., 2014; 81 Fronhofer \& Altermatt, 2015) that tracks, firstly, ecological dynamics, such as spatial spread in a linear 82 landscape, population densities as well as dispersal events, and, secondly, evolutionary changes, more 83 specifically the evolution of dispersal and the concurrent evolution of reproductive and competitive ability.

84 In each replicate linear landscape, populations are initialized at one end of the landscape and individuals 85 may subsequently spread following a stepping stone model (nearest neighbor dispersal).

${ }_{86}$ We assume local competition for resources and, for simplicity, non-overlapping generations. As a result 
of standing genetic variation present in the beginning and of subsequent mutations, the distribution of traits in a population may shift, leading to evolutionary changes in dispersal. Since it is well known that dispersal is costly (Bonte et al., 2012), we assume that more dispersive individuals reproduce less due to their investment of energy into dispersal (Fronhofer \& Altermatt, 2015) (dispersal-fecundity tradeoff; Eq. 4). Furthermore, reproduction and competitive ability are positively correlated (Eq. 3) due to underlying consumer-resource dynamics (Matessi \& Gatto, 1984) (detailed derivation in the Supporting Information).

In addition to a control scenario (scenario 1), in which a range expansion occurs into a previously empty landscape, we implemented scenarios that include linearly increasing spatial gradients in local mortality (scenarios 2 and 3). We contrast a setting in which dispersal propensity may evolve and the organisms do not have the capacity to sense the environmental change in such a gradient (scenario 2) and a scenario in which we assume that individuals have perfect information to make an optimal dispersal decision plastically and therefore evolutionary changes become irrelevant (scenario 3). Informed dispersal is based on a cost-benefit analysis, which takes into account population densities (i.e., competition) in the patch of origin and in all potential target patches, as well as the effect of the mortality gradient.

The model was designed to be as simple as possible and to provide qualitative predictions on the impact of environmental gradients and information use on the ecological and evolutionary dynamics of range expansions. We therefore ran an extensive sensitivity analysis (Figs. S6 - S10). We neither parametrize nor fit the model to the experimental data.

\section{Landscape and the environmental gradient}

For simplicity, we assume a linear landscape of 100 interconnected patches. At the start of each replicate simulation only the first five patches are populated. The landscape allows individuals to disperse following a stepping stone model, that is, we assume nearest neighbor dispersal with reflecting boundary conditions at both ends of the landscape. In scenarios 2 and 3, which include an environmental mortality gradient, we assume that this additional source of local mortality $\left(\mu_{x}\right)$ acts after reproduction and density regulation (see below) and before dispersal. The mortality gradient is linear and increases from $\mu_{1}=0$ in the first patch to $\mu_{100}=1$ mortality in the last patch.

\section{Dispersal}

Besides being governed by the landscape setting as described above, dispersal of individuals is assumed to be either genetically controlled (scenarios 1 and 2) or fully plastic and informed (scenario 3 ). We here only describe the former two scenarios, the latter will be dealt with in detail below. The probability of dispersing, more specifically emigrating from a natal patch, is genetically controlled by a haploid locus 
that codes for the dispersal rate $\left(d_{i}\right)$. When an individual $(i)$ disperses according to its specific dispersal rate, the direction in the linear landscape (i.e., towards the range core or towards the range front) is drawn randomly.

We do not assume explicit dispersal costs (Bonte et al., 2012). However, dispersal is implicitly costly, as we assume that dispersal trades off with reproduction and competitive ability as described below.

\section{Reproduction and density regulation}

Reproduction occurs after dispersal and follows a modified logistic, density-dependent growth model based on Beverton \& Holt (1957):

$$
N_{x, t}=N_{x, t-1} \lambda \frac{1}{1+\alpha N_{x, t-1}}
$$

where $N_{x, t}$ is the population size in patch $x$ at time $t, \lambda$ is the growth rate and $\alpha$ the intra-specific competition coefficient as introduced above. As reproductive $\left(\lambda_{i}\right)$ and competitive ability $\left(\alpha_{i}\right)$ are individualbased traits, the mean number of offspring an individual produces at time $t$ in a population of size $N_{x, t}$ is:

$$
\Lambda_{i}=\lambda_{i} \frac{1}{1+\sum_{j=1}^{j=N_{x, t}} \alpha_{j}} .
$$

We include demographic stochasticity by assuming that reproduction follows a Poisson process and drawing the realized number of offspring for individual $i$ from a Poisson distribution with mean $\Lambda_{i}$. After reproduction all individuals of the previous generation die.

\section{Trait correlations and trade-offs}

As outlined in the Supporting Information ("Linking consumer-resource dynamics to logistic growth"), we assume that reproductive and competitive ability $\left(\lambda_{i}\right.$ and $\alpha_{i}$, respectively) are individual-based traits that correlate positively:

$$
\alpha_{i}=\alpha_{0} \lambda_{i}^{\rho}
$$

with $\alpha_{0}$ as a baseline competitive ability and $\rho$ as the correlation exponent between competitive and reproductive ability. As Fronhofer \& Altermatt (2015) showed previously, a large part of changes in competitive ability seem driven by changing feeding rates and not by changing assimilation coefficients. We therefore assume $\rho=2$ as a standard scenario following the logic outlined above. For a summary of parameters and tested values refer to Tab. S1.

Furthermore, we assume that dispersal is costly (Bonte et al., 2012) and trades off with reproduction, 
and, therefore, also with competitive ability:

$$
\lambda_{i}=\lambda_{0} e^{d_{i} \tau}
$$

where $\lambda_{0}$ is the baseline fecundity, $d_{i}$ the dispersal rate of individual $i$ and $\tau$ the strength of the trade-off between dispersal and fecundity.

\section{Information use}

In scenario 3 we assume that dispersal is plastic in the sense that individuals make informed dispersal decisions. The decision of whether to disperse to one of the two neighboring patches in the linear landscape or to stay in the natal patch is based on a cost-benefit calculation. We assume that individuals have perfect knowledge on the patch densities in their natal patch $\left(N_{x, t}\right)$ and in the potential target patches, as well as information on local mortality $\left(\mu_{x}\right)$ due to the mortality gradient. Individuals disperse to the patch $x$ that maximizes their fitness according to Eq. 2:

$$
\Lambda_{i, x}=\lambda_{i} \frac{1}{1+\sum_{j=1}^{j=N_{x, t}} \alpha_{j}}\left(1-\mu_{x}\right)
$$

This approach only accounts for direct fitness benefits and ignores inclusive fitness (Hamilton \& May, 1977). Our simulations therefore underestimate dispersal and spatial spread rates in the informed scenario. For a detailed treatment of the effect of kin competition on range dynamics see Kubisch et al. (2013).

\section{Evolution and the genetic algorithm}

Evolutionary dynamics are an emergent phenomenon of any individual-based model that allows for variation in heritable, individual-based traits. The specific simulation scenario leads to selection pressures, such as spatial selection (Phillips et al., 2010; Shine et al., 2011) in range expansion scenarios, for instance. We here assume that dispersal rate $\left(d_{i}\right)$, fecundity $\left(\lambda_{i}\right)$ and competitive ability $\left(\alpha_{i}\right)$ are heritable and passed on from parent to offspring with a mutation rate $m=0.001$ that leads to a random change of the trait value drawn from a Gaussian distribution with mean zero and standard deviation $\Delta m=0.1$. The only trait subject to mutations is the dispersal trait $\left(d_{i}\right)$ since both fecundity $\left(\lambda_{i}\right)$ and competitive ability $\left(\alpha_{i}\right)$ depend on dispersal via the trade-off and correlation structures explained above (Eq. 3 and $4)$.

At the genotype level we do not implement any boundary conditions on the dispersal trait, that is, depending on mutations $d_{i}$ may be negative or $>1$. At the phenotype level values $<1$ are set to zero 
and values $>1$ are set to 1 . These phenotypic values are are also used to calculate fecundity according to Eq. 4.

\section{Simulation experiments}

All simulations were initialized with populations at a baseline equilibrium density $\left(\frac{\lambda_{0}-1}{\alpha_{0}}\right)$ in the first five patches in order to allow the individuals to subsequently spread through the landscape. Individuals in these populations were initialized with random dispersal rates $\left(0 \leq d_{i} \leq 1\right)$ as standing genetic variation. All simulations were allowed to proceed for 95 generations which, given the stepping stone dispersal model, is the minimal time span needed to reach the opposite end of the landscape. In general, simulations were replicated 20 times. The sensitivity analysis of scenario 3 (gradient and information) was performed on less replicates (between 1 and 10) as these simulations show only very little variation between replicates (see Fig. 1 E) and take an excessive amount of time to run. Please see Tab. S1 for tested parameter combinations and Figs. S6 - S10 for a sensitivity analysis.

\section{Microcosm experiments}

\section{Study organism}

We used Tetrahymena pyriformis, a unicellular freshwater ciliate, as a model organism (Altermatt et al., 2015; Fronhofer \& Altermatt, 2015). Tetrahymena pyriformis is small (approx. $40-50 \mu \mathrm{m}$ along the major axis), has a relatively short doubling time (approx. $4-5 \mathrm{~h}$ ) and reaches high densities (equilibrium densities: 5,000 - 15,000 individuals/mL) which makes it well suited for ecological and evolutionary experiments (Altermatt et al., 2015). We kept T. pyriformis under controlled environmental conditions at $20^{\circ} \mathrm{C}$ in protist pellet medium $(0.46 \mathrm{~g} / \mathrm{L}$; Carolina Biological Supply) with bacteria ( 5 vol- $\%$ of standardized 7-day-old cultures of Serratia fonticola, Brevibacillus brevis and Bacillus subtilis) as food resources. We used the same protist cultures as Fronhofer \& Altermatt (2015) and therefore started evolution experiments with standing genetic variation. The cultures were originally obtained from Carolina Biological Supply and regularly restocked to conserve genetic variation (Cadotte, 2007).

\section{Microcosm landscapes}

The range expansion experiments were performed in linear landscapes consisting of 14 interconnected microcosms (patches). We used $20 \mathrm{~mL}$ vials (Sarstedt), connected them with silicone tubing (VWR; $4 \mathrm{~mm}$ inside diameter) and a stopcock (B. Braun Discofix) to regulate dispersal (length of tubing and stopcock: $6 \mathrm{~cm}$ ). All experiments were replicated 6 times in two experimental blocks of 3 replicates each separated by 1 day. 


\section{Scenarios and experimental procedure}

At the beginning of each experiment, the first patch of a landscape was filled with a week-old Tetrahymena pyriformis culture that had reached its equilibrium density. Subsequently, the stopcocks were opened and dispersal was allowed for 4 hours. In order to avoid aging of medium and to limit contaminations, the landscape was not completely filled with medium from the start of the experiment, but empty patches were added subsequently to the landscape front. At the beginning of the experiment, 3 of the 14 patches were filled. At each day of the experiment, one additional patch filled with freshly bacterized medium (5 vol-\%) was added at the front. Since all patches between range core and range front were connected, dispersal could potentially occur across multiple patches and towards the range front as well as towards the range core.

To analyze the influence of information use on the eco-evolutionary dynamics of range expansions into environmental gradients, we designed two experimental treatments in addition to the control treatment (scenario 1) described above. For both, uninformed (scenario 2) and informed scenarios (scenario 3) a linear mortality gradient was applied, ranging from $0 \%$ mortality in the first patch to $100 \%$ mortality in the last patch. In the uninformed scenario (scenario 2), depending on the mortality gradient, a certain volume of the microcosm was removed, discarded and replaced with bacterized medium. In the informed scenario (scenario 3), we followed the same procedure but replaced the volume with dead T. pyriformis from a 4-days old culture that was killed by ultrasonication (duration: 4 min.; amplitude: 40\%; pulse on: 2 sec.; pulse off: 1 sec; ice bath to avoid heating). We therefore use dead T. pyriformis and their chemical cues to inform the protists in the experiments about the increasing mortality in the landscape. Previous to the experimental evolution assays we performed chemical orientation assays to confirm that dead conspecifics are indeed used as a negative tactic cue (see Supporting Information "Effects of chemical cues provided by dead conspecifics").

The general experimental procedure was as follows: we first applied the respective treatments (scenario 1: control, scenario 2: mortality gradient, scenario 3: mortality gradient and information) and allowed for dispersal (4h) on one day. The following day allowed for regrowth. We therefore had discrete dispersal and growth phases in analogy to the individual-based model described above. In total, the evolution experiment took 26 days with 13 dispersal events and subsequently two days of common garden. Each scenario was replicated 6 times and the experimental units were arranged in two blocks of 3 replicates each shifted by one day due to the large number of samples to process. 


\section{Common garden and growth curves}

In order to tease apart plastic changes, due to environmental or parental effects, in dispersal (respectively, movement strategies), growth rates and competitive abilities from genetically or non-genetically inherited evolutionary changes, we transferred range core and range front populations to a common environment after the experimental evolution phase. We transferred all core and front populations from the end of the experiment to $200 \mathrm{~mL}$ Erlenmeyer flasks and added $100 \mathrm{~mL}$ freshly bacterized medium to the $15 \mathrm{~mL}$ from the experimental microcosms. This transfer reset all populations to roughly the same environmental conditions in terms of resource availability and chemical composition of the medium. After 2 days in this common environment, all populations were assessed for divergence in movement behaviour, growth rates and competitive abilities.

Growth rates and competitive abilities were estimated by performing growth curve experiments and subsequently fitting logistic growth curves (Eq. S2) to the time-series data. All growth curves were started with approx. 500 individuals per $\mathrm{mL}$ by diluting the populations from the common garden. 5 vol-\% bacteria from a standardized, 7-days old culture were added as resources. The growth of each population was followed for 10 days using video recording and analysis as described below.

Logistic growth curves were fitted to the individual replicates using a least-squares approach. Eq. S2 was solved (function 'ode' of the 'deSolve' package in $\mathrm{R}$ version 3.2.3) and the model was fit using the Levenberg-Marquardt algorithm (function 'nls.lm' of the 'minpack.lm' package) which minimizes the sum of squared residuals.

\section{Data collection}

Before a treatment was performed, a $0.5 \mathrm{~mL}$ sample of each patch was collected. In the control and uninformed scenario, the sampling volume was replaced with fresh, bacterized medium. In the informed scenario, the sampling volume was replaced with dead T. pyriformis and fresh, bacterized medium for the first patch, respectively.

A subsample was then used for video recording with a Leica M205 C stereomicroscope (16 fold magnification) and a Hamamatsu Orca Flash 4 video camera (imaged volume: $34.4 \mu \mathrm{L}$; sample height: $0.5 \mathrm{~mm}$ ). Videos of 20 seconds were recorded with a total of 500 gray scale images with a resolution of 2024 x 2024 pixels.

The general method of automated image analysis was introduced by Pennekamp \& Schtickzelle (2013); Pennekamp et al. (2015) and has successfully used in previous experiments (Giometto et al., 2014; Fronhofer et al., 2015b; Fronhofer \& Altermatt, 2015; Fronhofer et al., 2015a). The aim is to collect abundance data as well as morphological and behavioral data simultaneously and provide information at the indi- 
vidual level. The principle of automated image analysis first includes a cleaning step followed by different analytical steps to determine morphological traits (length, size), abundance and movement data (velocity, turning angle, Euclidean distance). The first step of the image analysis consists in identifying the objects of interest by segmenting the moving foreground from the static background. Therefore the difference between picture $t$ and $t+1$ was analyzed. In general, only particles with a size between 20 and 200 pixels and a minimal path length of 100 frames were included in the analysis. Trajectories of each individual were analyzed with the ImageJ MOSAIC plugin (Sbalzarini \& Koumoutsakos, 2005). Data of each sample (abundance, velocity, body size, turning angle) was saved as mean values. As previous work consistently showed that dispersal rates and movement behaviour correlate highly in these protist microcosms (Fronhofer \& Altermatt, 2015; Fronhofer et al., 2015a), we here use movement as a proxy for dispersal. Data can be downloaded from Dryad DOI: XXX.

\section{Statistical analysis}

Differences in velocity were analysed using linear mixed models (LMM). We included the experimental block (replicates 1-3 and 4-6) as a random effect in our analyses. We used a Gaussian error structure as the QQ-plots indicated that this assumption was not heavily violated. All analyses were performed at the population level, i.e. on mean parameters over all individuals in a sample. This approach is very conservative, since it significantly reduces the sample size given the high population densities and the individual-based data collected by video recording and analysis. These analyses were performed using $\mathrm{R}$ version 3.2.3 and the "lmerTest" package.

The distribution of population densities over space was compared between treatments using the empirical cumulative population density distributions (see Fig. S3 D-F). Again, we chose a very conservative approach and only compared the median cumulative density distributions of the treatments using the Cramer-von Mises (CvM) statistic $\left(\omega^{2}\right)$ for two samples. We therefore calculated the sum of the squared differences between two empirical cumulative density distributions $\left(\omega^{2}\right)$. We subsequently analysed significance levels by resampling (one-sided tests) and additionally provide Probability-Probability plots for visual analysis (Fig. S4). As we performed all pairwise comparisons (2 comparisons per treatment), we corrected the obtained significance thresholds using the Bonferroni method, which consists of multiplying the initially obtained significance thresholds with the number of comparisons.

The chemical orientation assay was analysed using generalized linear mixed models (GLMM) with binomial error distributions and counts of individuals choosing either the treatment or the control patch. We included "replicate" as a random effect to take into account the pairing between dispersal to control and treatment patches within one replicate. We further included a sample level random effect to account for overdispersion. 
The empirical correlation between competition coefficients $(\alpha)$ and growth rates $\left(r_{0}\right)$ for populations from the range core and the range margin was analysed using non-linear regressions (following Eq. 3) for grouped data with the function "nlsList" of the "nlme" package in $\mathrm{R}$ version 3.2.3. For this analysis, we only used data from scenarios 1 and 2 as we did not observe evolutionary dynamics in scenario 3 so that the classification into core and front populations is not meaningful. We nevertheless report data from scenario 3 in Fig. 3 B. Note that while the parameter estimates differ slightly if we nevertheless include scenario 3 in the analysis the confidence intervals clearly overlap for the parameter of interest $(\rho)$ implying no significant differences $\left(C I_{\text {core }(1,2)}^{\rho}: 1.80,2.11 ; C I_{\text {core }(1,2,3)}^{\rho}: 1.80,2.91 ; C I_{\text {front }(1,2)}^{\rho}: 4.31\right.$, $\left.7.86 ; C I_{\text {front }(1,2,3)}^{\rho}: 1.48,10.47\right)$.

\section{Results}

\section{Theoretical predictions}

In the control and gradient scenarios our theoretical analyses (Fig. 1) predict evolutionarily increased dispersal at the range front compared the range core (Fig. 1 B, D). However, the difference in evolved dispersal propensities between range core and front populations is reduced in the gradient scenario. Furthermore, we predict higher population densities at range fronts in the control scenario and, to a lesser extent, also in the gradient scenario (Fig. 1 A, C and S3 A, B). The invasion does not proceed as far in the gradient scenario as in the control, suggesting that a stable range border forms (Fig. 1 A, C and S3 A, B).

In the informed dispersal scenario, the density profile of populations across the range is inverted in comparison to the evolutionary scenarios implying lower densities at range fronts in comparison to range cores (Fig. 1 E and S3 C). These predictions qualitatively hold true across a large range of tested parameter values (Tab. S1; Figs. S6 - S10; especially for weak dispersal-fecundity trade-offs and fecunditycompetition correlation coefficients $>1$ ).

\section{Experimental range dynamics}

Our experimental results corroborate our theoretical predictions (Fig. 2). At the end of the range expansion phase we found increased movement velocities (which correlate strongly with dispersal (Fronhofer \& Altermatt, 2015; Fronhofer et al., 2015a)) at range fronts (Fig. 2 B, E, H), although the effect was weak in the informed scenario (control: LMM, space: $N=74(6), d f=72 t=11.79, p<0.001$; gradient: LMM, space: $N=77(6), d f=74 t=13.24, p<0.001$; information \& gradient: LMM, space: $N=64(6)$, $d f=62 t=4.69, p<0.001)$. After the common garden, the velocities in range core, respectively range 
front populations, were still significantly different in the control (Fig. 2 C; LMM, range position: $N=12$, $d f=9 t=3.94, p=0.0034$ ) and in the gradient scenario (Fig. $2 \mathrm{~F} ; \mathrm{LMM}$, range position: $N=12$, $d f=10 t=7.23, p<0.001$ ). No differences were observed in the informed scenario (Fig. 2 I; LMM, range position: $N=12, d f=10 t=-0.045, p=0.965)$.

Furthermore, we observed the predicted spatial distribution of population densities with high densities at range fronts and low densities in range cores in the control and gradient scenario (Fig. 2 A, C and S3 D, E). Information use completely inverted this pattern leading to significantly different distributions of population densities between informed and uninformed scenarios (Fig. 2, S3 D-F and Fig. S4) as well as to overall lower population densities.

\section{Concurrent changes in reproduction and competition}

At the end of the experiment, we measured population growth rates and competitive abilities after a common garden phase to separate genetic from plastic effects. We observed a positive correlation between growth rate and competitive ability (Fig. $3 \mathrm{~B}$ ), corroborating our assumption about this correlation (Fig. 3 A; for details see Eq. 3 and the Supporting Information). While individuals from range cores followed the theoretically predicted correlation quantitatively, individuals from range fronts shifted the predicted correlation curve towards increased growth rates (Fig. $3 \mathrm{~B}$ ).

\section{Discussion}

Research on range dynamics has often assumed homogeneous environments and consistently ignored that universally occurring environmental gradients provide information to spreading organisms about local conditions. This information may allow spreading organisms to plastically adapt their dispersal decisions, which can potentially alter macroecological patterns. We now theoretically and experimentally show that the ecological and evolutionary dynamics of species' ranges are not only driven by the direct, fitness relevant effect of environmental gradients but, most importantly, by the information content of such gradients.

We find that range expansions lead to increased dispersal at the range front in the control and gradient scenarios (Fig. 2 C, F), which is consistent with previous theoretical (reviewed in Kubisch et al., 2014), comparative (e.g., Thomas et al., 2001; Phillips et al., 2006; Lombaert et al., 2014) and experimental results (Fronhofer \& Altermatt, 2015). Importantly, however, the latter has hitherto only been studied in unrealistic environmentally homogeneous landscapes. The evolutionary increase in dispersal is due to spatial assortment and fitness advantages of dispersers that colonize empty habitat at the range front and therefore do not suffer from competition ("spatial selection" Phillips et al. 2010; Shine et al. 2011). In 
the gradient scenario, spatial selection is counteracted by increasing mortality. In the informed scenario, we find differences in dispersal only early during the range expansion phase, but not after the common garden (Fig. 2 H, I), which confirms our model assumption regarding complete plasticity of dispersal in this scenario.

Both, theoretical predictions and experimental results show a spatial density pattern of increasing population sizes towards the range front as long as no information is provided (Figs. 1, 2). These density patterns emerge in the theoretical results because more dispersive individuals at range fronts deplete resources less due to the trade-off between dispersal and reproduction (Eq. 4) and concurrent changes in competitive abilities (Eq. 3), which implies that patches at the range front can support higher equilibrium population densities (Fronhofer \& Altermatt, 2015). Our empirical results, especially the observed correlation between reproduction and competition (Fig. 3), support our model assumptions and the relationship between growth rate and competitive ability derived in the Supporting Information. In the informed scenario we do not find increased densities at the range front which is consistent with our finding that dispersal was completely plastic (Fig. 2 H, I), as the density differences are due to evolutionary dynamics. Thus, information use inverts the spatial distribution of population densities across a species' range.

Remarkably, in our experiments, the impact of the mortality gradient on the spatial distribution of densities was relatively weak, while the influence of information use was extremely strong in inverting the spatial pattern of population densities (Fig. 2 D, G and S3 D-F). This indicates that not the environmental gradient itself, but rather using information thereon drives range expansion dynamics into environmental gradients in our experimental system.

In our experiments, the mortality gradient selected for increased reproduction (Fig. 3 B). The quantitative difference between theoretical prediction and experimental results (Fig. S3) regarding the impact of information use can be linked to the shift in trait correlation structure we observed (Fig. 3). This shift in the correlation can be interpreted as the result of strong selection for high reproduction at range fronts, which explains why the effect of the mortality gradient was relatively small in the experiments (Fig. 2 D): Populations overcame increased mortality by increasing reproduction. The shift in the trait correlation structure is likely due to a change in foraging behavior from a linear to a saturating functional response (see Supporting Information) as reported previously (Fronhofer \& Altermatt, 2015).

We have here explored the eco-evolutionary consequences of an environmental gradient acting on mortality. Evidently, changes in local conditions can also act via reducing fecundity and previous theoretical work (e.g., Kubisch et al., 2010, 2016) has shown that the nature of the gradient may impact the ecoevolutionary dynamics of ranges (reviewed in Kubisch et al., 2014). Nevertheless, we here theoretically show that a combined gradient, which simultaneously increases mortality and decreases fecundity, does 
not alter our results qualitatively (Fig. S5). A pure fecundity gradient may, for instance, reduce or even cancel the increase in populations sizes we report across the range. However, we argue that information on environmental changes along the gradient will still be highly fitness-relevant and therefore readily used by organisms that are capable of plastically adjusting their dispersal strategy.

In conclusion, we show that environmental gradients have a two-fold effect consisting of 1) a direct fitness relevant effect of the gradients itself and 2) of the information the gradient conveys on the environmental change. This information can be used to steer dispersal decisions which has major consequences for the macroecological patterns of range expansions along environmental gradients. Informed dispersal does not only impact expansion dynamics but can completely invert the spatial distribution of population densities. Our theoretical and experimental findings highlight the need to include environmental heterogeneity and organisms' capacity to process information thereon into realistic predictions of invasion dynamics and range expansions.

\section{Acknowledgements}

We thank Mark van Kleunen for his input during the planning of experiments, Sebastian Schreiber for his literature suggestions and Anita Narwani, Jennifer William and Chelsea Little for feedback on an earlier version of the manuscript. Funding is from Eawag (to E.A.F.) and the Swiss National Science Foundation, Grant No. PP00P3_150698 (to F.A.).

\section{List of brief titles of items in the supplementary material}

1 Linking consumer-resource dynamics to logistic growth

2 Effects of chemical cues provided by dead conspecifics

3 Supplementary figures

4 Supplementary tables

\section{Biosketch}

We are part of the Institute of Evolutionary Biology and Environmental Studies at University of Zurich as well as the Department of Aquatic Ecology at Eawag. With our work we want to understand how species occur in space and time, how they interact, and how processes such as species invasions, dispersal or climate change affect natural communities. 


\section{Author contributions}

E.A.F., N.N. and F.A. designed the research; N.N. performed the experiments; N.N. and E.A.F. analysed the data; E.A.F. developed the stochastic modelling framework; E.A.F. and F.A. wrote the paper.

\section{Data accessibility}

All data and computer code will be archived in Dryad and the DOI will be included in the manuscript.

\section{References}

Altermatt, F., Fronhofer, E.A., Garnier, A., Giometto, A., Hammes, F., Klecka, J., Legrand, D., Mächler, E., Massie, T.M., Plebani, M., Pennekamp, F., Pontarp, M., Schtickzelle, N., Thuillier, V. \& Petchey, O.L. (2015) Big answers from small worlds: a user's guide for protist microcosms as a model system in ecology and evolution. Methods in Ecology and Evolution, 6, 218-231.

Beverton, R.J.H. \& Holt, S.J. (1957) On the dynamics of exploited fish populations. Chapman \& Hall, London.

Bonte, D., Van Dyck, H., Bullock, J.M., Coulon, A., Delgado, M., Gibbs, M., Lehouck, V., Matthysen, E., Mustin, K., Saastamoinen, M., Schtickzelle, N., Stevens, V.M., Vandewoestijne, S., Baguette, M., Bartoń, K., Benton, T.G., Chaput-Bardy, A., Clobert, J., Dytham, C., Hovestadt, T., Meier, C.M., Palmer, S.C.F., Turlure, C. \& Travis, J.M.J. (2012) Costs of dispersal. Biological Reviews, 87, $290-312$.

Burton, O.J., Pillips, B.L. \& Travis, J.M.J. (2010) Trade-offs and the evolution of life-histories during range expansion. Ecology Letters, 13, 1210-1220.

Cadotte, M.W. (2007) Competition-colonization trade-offs and disturbance effects at multiple scales. Ecology, 88, 823-829.

Clobert, J., Le Galliard, J.F., Cote, J., Meylan, S. \& Massot, M. (2009) Informed dispersal, heterogeneity in animal dispersal syndromes and the dynamics of spatially structured populations. Ecology Letters, 12, 197-209.

Dytham, C. (2009) Evolved dispersal strategies at range margins. Proceedings of the Royal Society BBiological Sciences, 276, 1407-1413.

Fronhofer, E.A. \& Altermatt, F. (2015) Eco-evolutionary feedbacks during experimental range expansions. Nature Communications, 6, 6844 . 
Fronhofer, E.A., Klecka, J., Melián, C. \& Altermatt, F. (2015a) Condition-dependent movement and dispersal in experimental metacommunities. Ecology Letters, 18, 954-963.

Fronhofer, E.A., Kropf, T. \& Altermatt, F. (2015b) Density-dependent movement and the consequences of the allee effect in the model organism Tetrahymena. Journal of Animal Ecology, 84, 712-722.

Giometto, A., Rinaldo, A., Carrara, F. \& Altermatt, F. (2014) Emerging predictable features of replicated biological invasion fronts. Proceedings of the National Academy of Sciences of the United States of America, 111, 297-301.

Hamilton, W.D. \& May, R.M. (1977) Dispersal in stable habitats. Nature, 269, 578-581.

Hastings, A., Cuddington, K., Davies, K.F., Dugaw, C.J., Elmendorf, S., Freestone, A., Harrison, S., Holland, M., Lambrinos, J., Malvadkar, U. \& et al. (2005) The spatial spread of invasions: new developments in theory and evidence. Ecology Letters, 8, 91-101.

Hill, J.K., Thomas, C.D. \& Huntley, B. (1999) Climate and habitat availability determine 20th century changes in a butterflys range margin. Proceedings of the Royal Society B-Biological Sciences, 266, $1197-1206$.

Holt, R.D. \& Barfield, M. (2011) Theoretical perspectives on the statics and dynamics of species' borders in patchy environments. American Naturalist, 178, S6-S25.

Holt, R.D., Keitt, T.H., Lewis, M.A., Maurer, B.A. \& Taper, M.L. (2005) Theoretical models of species' borders: single species approaches. Oikos, 108, 18-27.

Kelly, A.E. \& Goulden, M.L. (2008) Rapid shifts in plant distribution with recent climate change. Proceedings of the National Academy of Sciences of the United States of America, 105, 11823-11826.

Kubisch, A., Fronhofer, E.A., Poethke, H.J. \& Hovestadt, T. (2013) Kin competition as a major driving force for invasions. American Naturalist, 181, 700-706.

Kubisch, A., Holt, R.D., Poethke, H.J. \& Fronhofer, E.A. (2014) Where am I and why? Synthesising range biology and the eco-evolutionary dynamics of dispersal. Oikos, 123, 5-22.

Kubisch, A., Hovestadt, T. \& Poethke, H.J. (2010) On the elasticity of range limits during periods of expansion. Ecology, 91, 3094-3099.

Kubisch, A., Winter, A.M. \& Fronhofer, E.A. (2016) The downward spiral: eco-evolutionary feedback loops lead to the emergence of 'elastic' ranges. Ecography, 39, 261-269. 
Lombaert, E., Estoup, A., Facon, B., Joubard, B., Grgoire, J.C., Jannin, A., Blin, A. \& Guillemaud, T. (2014) Rapid increase in dispersal during range expansion in the invasive ladybird Harmonia axyridis. Journal of Evolutionary Biology, 27, 508-517.

Louthan, A.M., Doak, D.F. \& Angert, A.L. (2015) Where and when do species interactions set range limits? Trends in Ecology and Evolution, 30, 780792.

Matessi, C. \& Gatto, M. (1984) Does k-selection imply prudent predation? Theoretical Population Biology, 25, 347-363.

Melbourne, B.A. \& Hastings, A. (2009) Highly variable spread rates in replicated biological invasions: fundamental limits to predictability. Science, 325, 1536-1539.

Parmesan, C., Ryrholm, N., Stefanescu, C., Hill, J.K., Thomas, C.D., Descimon, H., Huntley, B., Kaila, L., Kullberg, J., Tammaru, T., Tennent, W.J., Thomas, J.A. \& Warren, M. (1999) Poleward shifts in geographical ranges of butterfly species associated with regional warming. Nature, 399, 579-583.

Parmesan, C. \& Yohe, G. (2003) A globally coherent fingerprint of climate change impacts across natural systems. Nature, 421, 37-42.

Pennekamp, F. \& Schtickzelle, N. (2013) Implementing image analysis in laboratory-based experimental systems for ecology and evolution: a hands-on guide. Methods in Ecology and Evolution, 4, 483-492.

Pennekamp, F., Schtickzelle, N. \& Petchey, O.L. (2015) Bemovi, software for extracting behavior and morphology from videos, illustrated with analyses of microbes. Ecology and Evolution, 5, 2584-2595.

Perkins, A.T., Phillips, B.L., Baskett, M.L. \& Hastings, A. (2013) Evolution of dispersal and life history interact to drive accelerating spread of an invasive species. Ecology Letters, 16, 1079-1087.

Phillips, B.L., Brown, G.P. \& Shine, R. (2010) Life-history evolution in range-shifting populations. Ecology, 91, 1617-1627.

Phillips, B.L., Brown, G.P., Webb, J.K. \& Shine, R. (2006) Invasion and the evolution of speed in toads. Nature, 439, 803-803.

Sagarin, R.D., Gaines, S.D. \& Gaylord, B. (2006) Moving beyond assumptions to understand abundance distributions across the ranges of species. Trends in Ecology and Evolution, 21, 524-530.

Sbalzarini, I. \& Koumoutsakos, P. (2005) Feature point tracking and trajectory analysis for video imaging in cell biology. Journal of Structural Biology, 151, 182-195. 
Sexton, J.P., McIntyre, P.J., Angert, A.L. \& Rice, K.J. (2009) Evolution and ecology of species range limits. Annual Review of Ecology, Evolution, and Systematics, 40, 415-436.

Shine, R., Brown, G.P. \& Phillips, B.L. (2011) An evolutionary process that assembles phenotypes through space rather than through time. Proceedings of the National Academy of Sciences of the United States of America, 108, 5708-5711.

Thomas, C.D., Bodsworth, E.J., Wilson, R.J., Simmons, A.D., Davies, Z.G., Musche, M. \& Conradt, L. (2001) Ecological and evolutionary processes at expanding range margins. Nature, 411, 577-581.

Williams, J.L., Snyder, R.E. \& Levine, J.M. (2016) The influence of evolution on population spread through patchy landscapes. American Naturalist, 188, 15-26.

\section{Figure Legends}

Figure 1: Range dynamics - theoretical predictions. (A - B) Expansion into homogeneous environment (control). Population densities increase from range core to front due to dispersal-fecundity trade-offs (Eq. 4) and fecundity-competition correlations (Eq. 3). Spatial selection leads to increased dispersal at range fronts. (C - D) Expansion into a mortality gradient. Density patterns are not fundamentally altered during a major part of the expansion (see also Fig. S3). However, increasing mortality locally reduces population densities and selects against dispersal. (E - F) Expansion in a mortality gradient and information use. Dispersal is plastic and individuals are fully informed about the mortality gradient, population densities in their natal and potential target patches (Eq. 5). The distribution of population densities over space is inverted (Fig. S3). Dispersal does not evolve, but it is predicted to be plastically higher at the range front during the expansion due to the decision rule. Temporal snapshots: $t=[10,30,50,70,90]$. Parameter settings: $\lambda_{0}=14, \alpha_{0}=0.00001, \rho=2, \tau=2$. We report medians over 20 replicate simulations (solid line; blue (range core) to red (range front)) and the 25th and 75th percentiles (grey shading; darker with time).

Figure 2: Range dynamics - experimental results. As predicted, the spatial distribution of population densities (A, D, G) showed an increase in densities towards the range front in the control (A) and gradient (D) scenarios. Information use (G) inverted this pattern (see Fig. S3 D-F; the distribution is statistically different from the other two; Fig. S4). On the last day of the evolution phase clear differences in movement over space was found in all scenarios $(\mathrm{B}, \mathrm{E}, \mathrm{H})$, although the effect was weak in the informed scenario. After the common garden phase, the velocities in range core respectively range front populations were still significantly different in the control $(\mathrm{C})$ and in the gradient scenario $(\mathrm{F})$. No 
differences were observed in the informed scenario (I). We report medians over 6 experimental replicates (solid line; blue (range core) to red (range front)) and the 25th and 75th percentiles (grey shading; darker with time). Stars indicate statistical significance (see text for details).

Figure 3: Concurrent evolution of reproduction and competition. (A) As derived in the Supporting Information, our model assumes a correlation between competitive ability $(\alpha)$ and fecundity $(\lambda ;$ Eq. 3$)$. Given a linear functional response we predict a roughly quadratic relationship $(\lambda-\alpha$ correlation coefficient $\rho=2$ ). Due to the trade-off between dispersal and fecundity, high fecundities and competitive abilities are predicted in the range core, where individuals are less dispersive (blue color tones indicate range core and red color tones range front; data from the control scenario; see Fig. 1). (B) Empirically measured competition coefficients $(\alpha)$ and growth rates $\left(r_{0}\right)$ after the common garden phase. The theoretically predicted relationship between competition and reproduction was found for core populations (blue; empirically measured $\alpha_{0}=0.0074$ (CI: 0.0053, 0.0094); $\rho=1.96$ (CI: 1.80, 2.11); only data form scenarios 1 and 2). However, selection acting during the range expansion altered this relationship (red; $\alpha_{0}=11.24$ (CI: $-29.11,51.60) ; \rho=6.09$ (CI: 4.31, 7.86); only data form scenarios 1 and 2) allowing individuals at the range front to have higher reproductive rates than theoretically predicted. Increased reproduction is highly advantageous as populations at the range front experience strong selection for both dispersal and reproductive ability. 
Figures
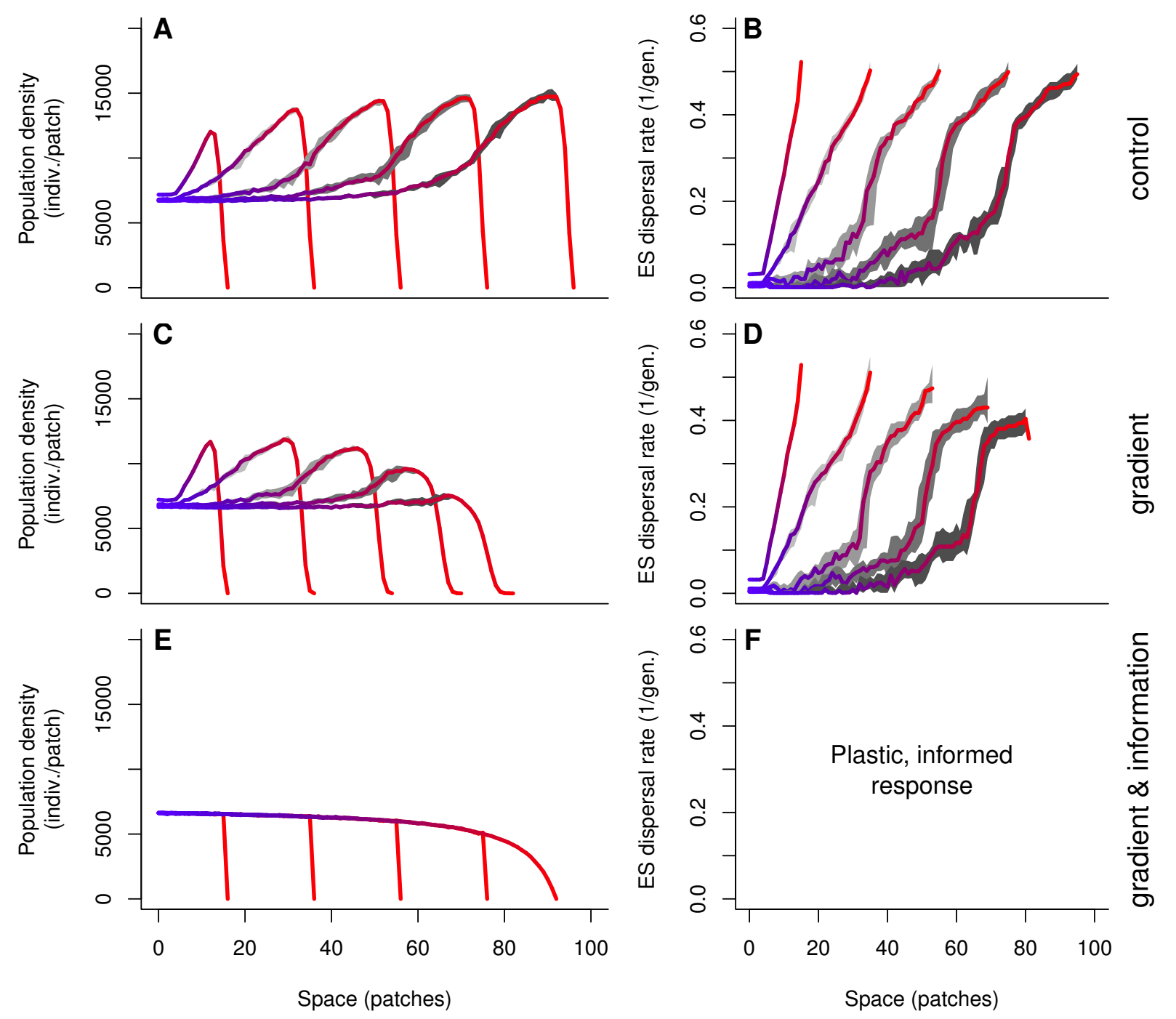

Figure 1 

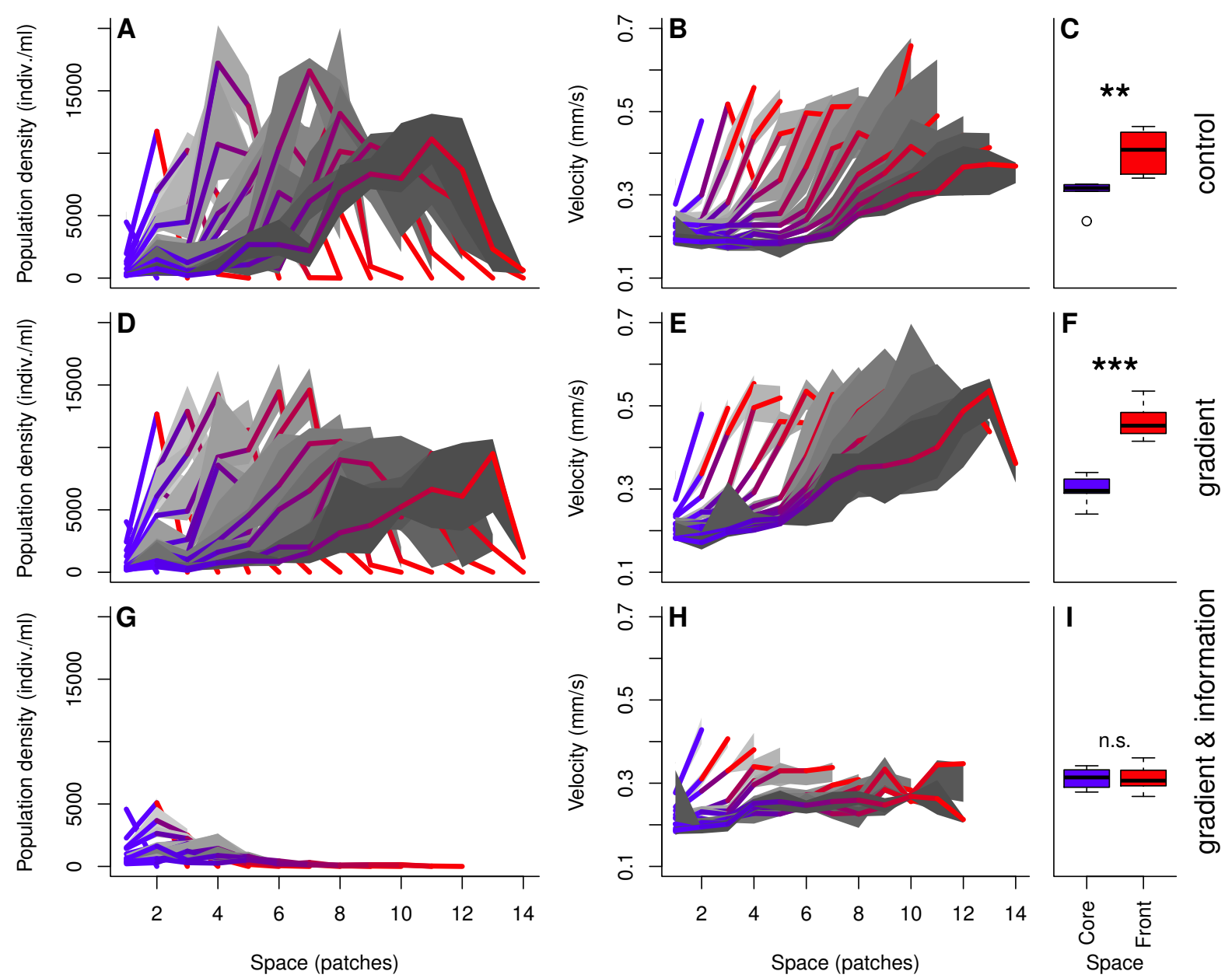

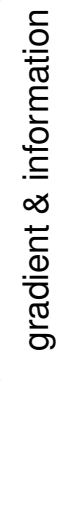

Figure 2 

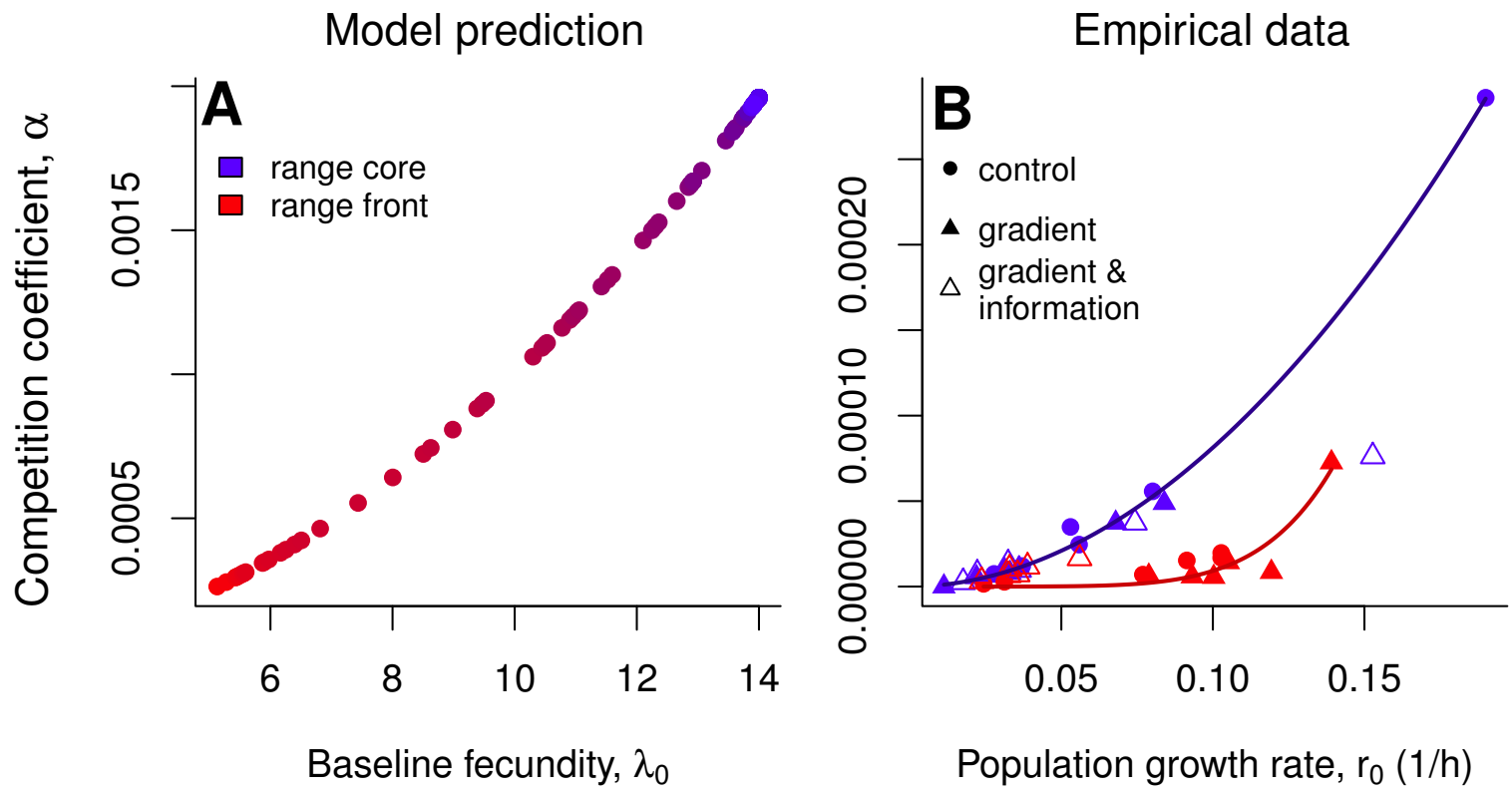

Figure 3 